CARDIOVASCULAR MEDICINE

\title{
A national programme for on-site defibrillation by lay people in selected high risk areas: initial results
}

\author{
C S Davies, M C Colquhoun, R Boyle, D A Chamberlain
}

Heart 2005;91:1299-1302. doi: 10.1136/hrt.2003.032631

See end of article for authors' affiliations

....................

Correspondence to: Dr Michael C Colquhoun

Wales Heart Research Institute, College of Medicine, University of Cardiff, Heath Park, Cardiff CF14 4XN, UK; colquhoun@bishopsfrome. u-net.com

Accepted 1 December 2004
Objective: To report on the effectiveness of an initiative to reduce deaths from sudden cardiac arrest occurring in busy public places.

Setting: 110 such places identified from ambulance service data as high risk sites.

Patients: 172 members of the public who developed cardiac arrest at these sites between April 2000 and March 2004. 20592 defibrillator months' use is reported, representing one automated external defibrillator (AED) use every 120 months.

Intervention: 681 AEDs were installed; staff present at the sites were trained in basic life support and to use AEDs.

Main outcome measures: Initial rhythm detected by AED, restoration of spontaneous circulation, survival to hospital discharge.

Results: 172 cases of cardiac arrest were treated by trained lay staff working at the site before the arrival of the emergency services during the period. A shockable rhythm was detected in $135(78 \%)$, shocks being administered in 134 an estimated $3-5$ minutes after collapse; $38(28.3 \%)$ patients subsequently survived to hospital discharge. Spontaneous circulation was restored in five additional patients who received shocks but died later in hospital. In 37 cases no shock was initially indicated; one patient survived after subsequent treatment by paramedics, cardiopulmonary resuscitation having been given soon after collapse. Overall, irrespective of the initial rhythm, 39 patients (22.7\%), were discharged alive from hospital. For witnessed arrests of presumed cardiac cause in ventricular fibrillation (an international Utstein comparator) survival was 37 of 124 (29.8\%).

Conclusions: The use of AEDs by lay people at sites where cardiac arrest commonly occurs is an effective strategy to reduce deaths at these sites.
$\mathrm{S}$ trategies to reduce deaths from sudden cardiac arrest depend crucially on reducing the interval from the onset of a potentially lethal arrhythmia to definitive treatment, particularly attempted defibrillation in appropriate cases. ${ }^{1}$ In the UK, as in most countries, the response times of conventional emergency ambulance services are too long for effective treatment to be available for most victims. The possibility of successful resuscitation declines by about $23 \%$ with every additional minute of response time. ${ }^{2}$ Recent audit figures from south east England (population 20 million) show that fewer than $2 \%$ of such victims attended by ambulance service personnel survive to leave hospital. ${ }^{3}$

Training lay people to use automated external defibrillators (AEDs) is one strategy to reduce the delay in providing defibrillation. Modern AEDs are safe, reliable, inexpensive, and simple to use, and they require little maintenance. Their potential value has been shown convincingly. Particularly impressive results have been reported from casinos in the USA with a survival rate of $74 \%$ when defibrillation was carried out within three minutes of a witnessed collapse, a delay rarely achievable by conventional emergency medical services but setting a standard. ${ }^{4}$ Other encouraging reports to date have come from other high risk locations including an airport and sporting venues, or have concerned specific groups of individual responders such as airline cabin staff or police officers whose occupation includes a duty to respond to emergencies. ${ }^{5-9}$

The treatment of sudden cardiac death from ventricular fibrillation (VF) by lay people trained to use AEDs was established in England as a part of a National Health Service provision in 1999. ${ }^{10}$ Audit of this innovative national programme was required by the government at the outset to assess the effectiveness of the project and to help determine future investment in the strategy. This paper is based on the prospective audit in place from the outset and reports the results of the initial stage of the project, the first national programme of its type in the world.

\section{METHODS}

The details of this programme and its implementation have been described previously. ${ }^{11}$ Briefly, AEDs were located in busy public places identified from routine ambulance service data as sites where cardiac arrest commonly occurred. Staff who worked at these sites volunteered to be trained in basic life support and the use of an AED in classes lasting four hours. The training procedures are described elsewhere; briefly, a standardised competency based curriculum with adequate manikin practice is taught at each location, supplemented by the use of simulated cardiac arrest scenarios. At sites outside London, training is provided by the statutory ambulance services. In London, trainers from St John Ambulance undertake the classes.

AEDs are located in unlocked protective cabinets at the selected locations in a density that ensures that equipment is available within a notional two minutes' walk from any part of the premises to which the public have access.

Data about each resuscitation attempt are recorded at the location in a format compatible with the Utstein system for uniform recording of pre-hospital resuscitation attempts. ${ }^{12}$ Experience gained during this phase of auditing the programme led to the design of the report form and audit

Abbreviations: AED, automated external defibrillator; CPR, cardiopulmonary resuscitation; $\mathrm{VF}$, ventricular fibrillation 
methods described in detail elsewhere. ${ }^{13}$ The intervals between collapse and the institution of basic life support and defibrillation are usually estimated but are sometimes supported by data from ambulance despatch centres or other control systems. The data on the report form are analysed in conjunction with a download of data stored electronically in the memory chip in the AED, so that both the ECG and the procedures undertaken during each event can be analysed in detail.

Information about ambulance response times at Heathrow Airport was obtained from the London Ambulance Service so that times to defibrillation by ambulance personnel and by lay responders at this location could be approximately compared. Heathrow Airport provided more cases for this study than any other single site. Further information about response times to arrests occurring at other sites is sometimes made available to the Department of Health by the service concerned.

\section{RESULTS}

Of 692 AEDs procured for the initial phase of the project, 681 were deployed for use and the remaining 11 were held in reserve as spares in case of machine malfunction, damage, or theft.

Ambulance service data indicated overwhelmingly that the most common public sites for cardiac arrest to occur were transport facilities, particularly airports and mainline railway stations. Accordingly, the 681 AEDs were distributed to 110 sites as follows: 300 AEDs at 23 airports, 267 at 66 main line rail stations, 12 at four major bus stations, eight at two ferry terminals, nine at a single shopping complex, and 85 at 14 major London Underground stations. AEDs were deployed over 32 months from April 2000 to November 2002 as suitable sites were identified and personnel were trained.

The experience reported here comprises 20592 defibrillator months at the 110 sites with 172 resuscitation attempts by the end of March 2004. This represents one AED use every 120 defibrillator months. One hundred and fifty victims were men (average age 63.4 years), 21 were women (average age 61.2 years), and in one case sex was not recorded. The cardiac arrest was witnessed in 157 (91\%) cases and unexpected in all but one case where staff placed the AED on a conscious passenger who appeared unwell. Sinus rhythm was recorded initially but he lost consciousness with the onset of ventricular tachycardia/VF soon afterwards. In the 15 cases where the collapse was not witnessed, the initial rhythm was VF in 11, profound bradycardia in three, and not recorded in one. Ninety six arrests occurred in airports, 58 in railway stations, six in bus stations, two in ports or ferry terminals, five in the shopping centre, and five in underground railway stations.

Data about ambulance response times by the London Ambulance Service indicated that at Heathrow Airport the time to defibrillation would be considerably greater were AEDs not located at the airport ( $\mathrm{R}$ Donohue, M Damiani, personal communication). In 16 cases of cardiac arrest at Heathrow included in this report, only three were attended within eight minutes; the average response time was 12 minutes (median 10 minutes). In 63 further arrests occurring at 27 different sites equipped with AEDs (and attended by many different ambulance services), the average response time was 8.4 minutes (median 7 minutes).

\section{Exclusions}

During the period covered by this report, AEDs were taken to nine other cardiac arrests. In five of these, members of the emergency medical services took over the management of the arrest before the AEDs were deployed but trained volunteers participated in basic life support. Resuscitation was also attempted in four cases of prolonged cardiac arrest long after any prospects of success had passed. Two people had committed suicide while locked in public toilets and the third had had a cardiac arrest in an aircraft 90 minutes earlier. All were asystolic when found but revival was attempted before the precise circumstances of the events had been clarified. A fourth patient was found asystolic locked in a public toilet having collapsed from a suspected subarachnoid haemorrhage an unknown time beforehand. These cases are not included in the analysis.

\section{Principal analysis}

Of the 172 resuscitation attempts considered, a shockable rhythm was identified when the AED was attached in 135 $(78 \%)$ and a shock was delivered in 134; the machine failed in the other event. Thirty eight $(28.3 \%)$ of these patients subsequently survived to be discharged from hospital alive. In a further five cases spontaneous circulation was restored at the scene after the shocks but the patients later died in hospital. Shocks were administered from 3-5 minutes after collapse (medians of the individual estimated time intervals). For witnessed cardiac arrest of presumed cardiac cause with a shockable rhythm (an Utstein international comparator), 37 of $124(29.8 \%)$ patients survived to hospital discharge.

No shock was indicated after the initial analysis by the AED in the remaining 37 patients. The rhythm subsequently seen on the download was asystole in 13, profound bradycardia in 15, idioventricular rhythm in three, or sinus rhythm at a normal rate in three. No recording was available in three cases. Only one patient of the 37 (with an initial rhythm of asystole) survived; staff performed cardiopulmonary resuscitation (CPR) after the initial AED analysis advised that no shock was indicated. Paramedics who arrived later were able to restore the circulation after several doses of adrenaline resulted in VF, which responded to defibrillation. Spontaneous circulation was not restored at any stage in any of the other 36 cases.

Considering the whole group of 172 resuscitation attempts irrespective of the initial rhythm, 39 (22.7\%) patients survived to leave hospital alive. If the nine excluded cases are added, the survival rate for all arrests at the sites becomes $21.5 \%$. Thirty eight survivors were men with an average age (estimated in some) of 65.7 years. The oldest survivor was 91 and recovered fully after coronary bypass surgery.

\section{Limitations of the study}

Confidentiality rules within the UK have hampered the collection of follow up information because no official mechanism exists for obtaining information from hospitals. Patients who survive are generally aware of the events that led to their recovery, however, and we can request that they make themselves known when they learn of our interest; in other cases anonymised data can be obtained. Outcome data on patients who are not admitted alive to hospital are more readily obtained. Because we could not collect data systematically, our information on survival to hospital discharge may be incomplete (we were unable to obtain information about the outcome of two patients), but we believe that all patients with a successful outcome have been included.

In most cases the time intervals are estimated. While this method is known to have limitations, there is for practical purposes no other way of obtaining such data. Despite the limitations, the errors are unlikely to be great, particularly with the short time intervals in this series. They allow comparison with other series of resuscitation attempts where similar methods have been used.

We believe that we have included all cases of cardiac arrest that occurred at the sites during the study period. Staff complete a form each time a defibrillator is deployed, so the 
default position is to return a form regardless of the cause Cases of simple fainting or collapse due to causes other than cardiac arrest are evident from information on the report forms coupled with analysis of the download from data recorded by the AED. Similarly, we are not aware of any patients treated solely by the emergency services at any of the sites without the participation of the staff at the site. In five cases, however, emergency services were summoned by the public before staff present at the scene were aware of the arrest and reached the patient before the on-site responders. Such arrests are routinely reported by the staff, however.

\section{DISCUSSION}

This paper describes the outcome of the first phase of a government led national programme to provide AEDs in busy public places. The first people likely to respond to such a casualty-those working at the site-were trained to provide the definitive treatment for victims of cardiac arrest. The results testify to the effectiveness of this strategy at sites with a relatively high incidence of cardiac arrest. The report relates specifically to England because the four countries that constitute the UK have their own distinct arrangements for the provision of health care services. The decision to launch this national scheme was taken in 1999 before any evidence about the effectiveness of public access defibrillation schemes had been published and therefore was a bold move by the relevant government agencies.

Overall, $23 \%$ of all the patients survived to leave hospital alive, but the success rate was $28 \%$ among those where the AED interpreted the cardiac rhythm as potentially responsive to a shock-the group most likely to survive if defibrillated quickly. Furthermore, among witnessed arrests of probable cardiac cause with a shockable rhythm the success rate was almost $30 \%$. This is similar to data from other public access defibrillation schemes, although precise comparison is impossible because of operational differences, variations in the provision of bystander CPR, differences in the time delays in providing defibrillation, and differences in the characteristics of the rescuers or victims.

The success rate reported is due in part to the early application of all the crucial links in the chain of survival, particularly the relatively brief delay from collapse to defibrillation (most often performed an estimated 3-5 minutes after collapse). This was possible because the AED was situated near patients when they collapsed and was used by staff working nearby. No time was needed to make or process an emergency call or locate and dispatch a responder to the casualty. Few strategies that depend on travelling with the AED to reach the patient (such as conventional emergency medical services or lay first responders dispatched by ambulance control centres) can achieve comparable response times except on rare occasions.

For obvious reasons there is no direct comparator available to quantify precisely the effectiveness of this initiative. There is, however, strong circumstantial evidence that the rapid availability of defibrillation made a major impact on patient survival at the locations studied. We identify the following principal reasons for this.

Firstly, the rate of successful resuscitation reported in this series is much superior to that reported by ambulance services in England (around 2\%), which can only rarely match the response times reported here. ${ }^{3}$ The success rate reported is comparable with other situations in the UK where rapid defibrillation is carried out-for example, arrests witnessed by ambulance crews or patients defibrillated by general practitioners within four minutes of collapse. ${ }^{14}{ }^{15}$

Secondly, ambulance services in England aim at attending the majority $(75 \%)$ of life threatening calls within eight minutes, but ambulance response time is a poor surrogate for the time between collapse and defibrillation. It may take some minutes for the witness of an arrest to find a telephone and establish contact with ambulance control and the initial time processing the call is not included in the "response time". The response time ends with the arrival of the ambulance at the scene but the crew may take several minutes to reach the casualty (especially at large railway stations and airports), assess the victim, start resuscitation, and attach the AED. Enough epidemiological data exist to be certain that survival would be very much lower if the ambulance service were the sole provider of defibrillation, even if bystander CPR is performed, than the survival rate when lay people present at the scene attached the AED 3-5 minutes after collapse. Even with defibrillation eight minutes after collapse, studies indicate that the likely survival rate would at best be around $6 \% .^{2}$

Thirdly, the frequency of VF recorded in this series (135 of $172 ; 78 \%$ ) is very much higher than that reported in any ambulance series of resuscitation attempts. This is fully compatible with the rapid response time achieved by lay responders that enabled VF to be recorded more often before it had degenerated into apparent asystole.

Fourthly, information obtained about response times from ambulance services indicates a very much later time to defibrillation after their attendance.

Lastly, this report provides a striking comparison with historical data from the sites before AEDs were installed. Beforehand patients rarely, if ever, survived; afterwards more than one fifth of victims survived to hospital discharge.

In addition to on-site defibrillation schemes such as that reported here, AEDs are also used in the UK by lay people who act in first responder schemes. These are often based in remote rural areas where trained volunteers despatched by ambulance control centres travel to potential victims because the volunteers are likely to reach the victim more quickly than a conventional ambulance would. The two strategies should be regarded as complementary because they serve different types of populations at risk. Careful audit of public access defibrillation schemes such as that presented here is required not only to safeguard clinical standards but also to provide the basis to guide future policies. Some have questioned the effectiveness of situating AEDs in public places, but the doubts rested on theoretical data based on local experience. ${ }^{16}$ It has been suggested that expansion of public access defibrillation be blocked until the outcome from randomised controlled trials of the use of AEDs in public places is available. ${ }^{17}$ Such trials could not likely be performed in the UK. The evidence presented testifies adequately to the effectiveness of these schemes, certainly for the people who sustain a cardiac arrest at such places. There is little evidence about the value of the alternative first responder strategy from the UK, but initial evidence suggests that substantially fewer patients are resuscitated because of the longer response intervals generally achieved by these schemes. ${ }^{18}$

This present report is based on data from cardiac arrests at many sites in England and fully supports the growing evidence from several centres in the USA for the effectiveness of deploying AEDs at selected high risk sites. The initial data reported here are encouraging and support the establishment of public access defibrillation as a core part of health service provision.

\section{Conclusions}

The deployment of AEDs in busy public places and use by trained responders working at the site is an effective strategy for treating one specific group of patients experiencing outof-hospital cardiac arrest. The reported scheme placed AEDs at sites where cardiac arrest was known to occur most commonly. This is a justifiable strategy to deal with a 
predictable event at a site where there is a duty of care to the clients and customers present. This initial experience from sites with the highest frequency of cardiac arrest is encouraging but only further experience can determine the effectiveness of AEDs used by lay people at other sites or deployed in other systems.

\section{ACKNOWLEDGEMENTS}

We thank Rachael Donaghue and Michael Damiani of London Ambulance Service for information on response times, and Anna Oakley and Helen Williams of the Department of Health for help with data collection.

\section{Authors' affiliations}

C S Davies, R Boyle, Department of Health, London, UK

M C Colquhoun*, D A Chamberlain*, Wales Heart Research Institute,

College of Medicine, University of Cardiff, Cardiff, UK

*Also the Prehospital Emergency Research Unit, Lansdowne Hospital, Canton, Cardiff, UK

\section{REFERENCES}

1 Anon. Guidelines 2000 for resuscitation and emergency cardiovascular care The automated external defibrillator a key link in the chain of survival. Resuscitation 2000:46:73-6.

2 De Maio VJ, Steill IG, Wells GA, et al. Optimal defibrillation response intervals for maximum out of hospital cardiac arrest survival rates. Ann Emerg Med 2003;42:242-50

3 Cave L, Evans L. Personal communication on behalf of the South East Ambulance Clinical Audit Group ( SEACAG).
4 Valenzuela TD, Roe D, Nichol G, et al. Outcomes of rapid defibrillation by security officers after cardiac arrest in casinos. N Engl J Med 2000;343:1206-9.

5 Caffrey S, Willoughby PJ, Pepe PE, et al. Public use of automated external defibrillators. N Engl J Med 2002;347:1242-7.

6 Wassertheil J, Keane G, Fisher N, et al. Cardiac arrest at the Melbourne Cricket Ground and Shrine of Remembrance using a tired response strategy: a forerunner to public access defibrillation. Resuscitation 2000;44:97-104.

7 O'Rouke M, Donaldson E. Management of ventricular fibrillation in commercial airliners. Lancet 1995;345:515-6.

8 Page RL, Joglar JA, Kowal RC, et al. Use of automated external defibrillators by a US airline. N Engl J Med 2000;343:1210-6.

9 White RD, Vukov LF, Bugliosi TF. Early defibrillation by police: initial experience with measurement of critical time intervals and patient outcome. Ann Emerg Med 1994;23:1009-13.

10 Department of Health. Saving lives: our healthier nation. London: HMSO, 1999.

11 Davies CS, Colquhoun M, Graham S, et al. Public access defibrillation: the establishment of a national scheme for England. Resuscitation 2002;52:13-21.

12 Chamberlain DA, Cummins RO, Abrahamson NS, et al. Recommended guidelines for uniform reporting of data from out-of-hospital cardiac arrest: the 'Utstein style.' Resuscitation 1991;22:1-26.

13 Colquhoun M, Davies CS, Harris, et al. Public access defibrillation: designing a universal report form and database for a national programme. Resuscitation 2004;61:49-54.

14 Sedgewick ML, Dalziel K, Watson J, ef al. The results of the second year of the Heartstart Scotland project in the Utstein style. Resuscitation 1993;26:75-88.

5 Colquhoun MC. Defibrillation by general practitioners. Resuscitation 2002;52:143-8.

16 Pell JP, Sirel JM, Marsden AK, et al. Potential impact of public access defibrillators on survival from cardiac arrest: retrospective cohort study. BMJ 2003;325:515-7.

17 Pell JP. The debate on public place defibrillators: charged but shockingly ill informed. Heart 2003;89:1375-6.

18 Colquhoun M, Davies CS, Woollard M, et al. A comparison of different strategies for public access defibrillation. 6th Congress of the European Resuscitation Council, Firenze, Italy, October 2002. Resuscitation 2002;55: 115.

\title{
IMAGES IN CARDIOLOGY
}

\section{Coronary artery stenosis caused by an aortic root abscess}

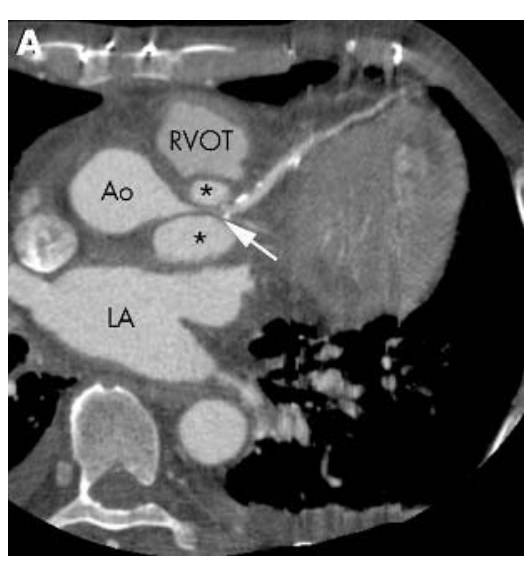

\begin{abstract}
73 year old man with active endocarditis and high grade valvar aortic stenosis underwent biological valve replacement. His postoperative course was complicated by persistent fever and delayed recovery. Two months later he presented with chest pain, dyspnoea, and ST segment elevation on the ECG suggesting myocardial ischaemia. An electrocardiographically gated, isotropic 16 slice cardiac computed tomography (CT) examination demonstrated a destructed subvalvar portion of the left ventricular outflow tract with a leakage feeding a perivalvar cavity (panels A and B, asterisks: Ao, aorta; LA, left atrium; LV, left ventricle; PA, pulmonary artery; RVOT, right ventricular outflow tract). This cavity encircled and compressed the main branch of the left coronary artery (panels A and B, arrow). Thus, the patient underwent immediate surgery with resection of a paravalvar abscess and reconstruction of the left ventricular outflow tract.

Paravalvar abscess is an often fatal complication of aortic valve endocarditis. Thus, early diagnosis and treatment are of utmost importance. However, even transoesophageal echocardiography can miss an abscess. The case presented here demonstrates the usefulness of the latest CT technology for depicting a paravalvar abscess. The main advantages of isotropic CT are the wide field of view and the multiplanar viewing capabilities without restriction to specific imaging windows, which makes it attractive as an additional imaging method in patients with suspected endocarditis but persistent diagnostic uncertainty.
\end{abstract}
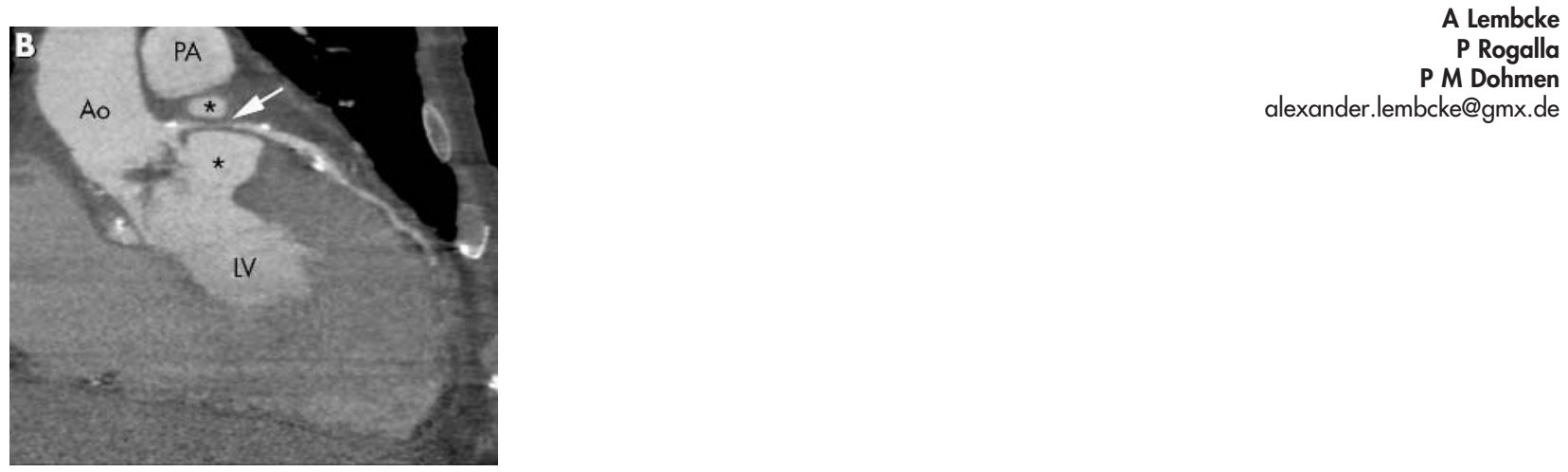